\title{
Gravitational decoupling, hairy black holes and conformal anomalies
}

\author{
Pedro Meert ${ }^{1, \mathrm{a}}$, Roldao da Rocha $^{2, \mathrm{~b}}{ }_{(\mathbb{C})}$ \\ ${ }^{1}$ Center of Physics, Universidade Federal do ABC, 09210-580 Santo André, Brazil \\ ${ }^{2}$ Center of Mathematics, Federal University of ABC, 09210-580 Santo André, Brazil
}

Received: 15 September 2021 / Accepted: 11 February 2022 / Published online: 24 February 2022

(C) The Author(s) 2022

\begin{abstract}
Hairy black holes in the gravitational decoupling setup are studied from the perspective of conformal anomalies. Fluctuations of decoupled sources can be computed by measuring the way the trace anomaly-to-holographic Weyl anomaly ratio differs from the unit. Therefore the gravitational decoupling parameter governing three hairy black hole metrics is then bounded to a range wherein one can reliably emulate AdS/CFT with gravitational decoupled solutions, in the tensor vacuum regime.
\end{abstract}

\section{Introduction}

Gravitational decoupling methods comprise established successful protocols used to generate analytical solutions of the Einstein's effective field equations [1-6]. The gravitational decoupling and some extensions were studied in Refs. [4,7-15] and have been applied to kernel solutions of general relativity to construct new physically realistic solutions that describe stellar distributions, including anisotropic ones [16-32]. References [33-35] derived accurate physical constraints on the parameters in gravitational decoupled solutions, using the WMAP, eLISA and LIGO. The gravitational decoupling procedure iteratively constructs, upon a given isotropic source of gravitational field, anisotropic compact sources of gravity, that are weakly coupled. One starts with a perfect fluid, then coupling it to more elaborated stressenergy-momentum tensors that underlie realistic compact configurations [36-56].

Any action related to a classical conformal theory is invariant under Weyl transformations. Since the variation of the action with respect to the background metric is proportional to the stress-energy-momentum tensor, then the variation of the action with respect to a conformal rescaling is pro-

\footnotetext{
a e-mail: pedro.meert@ufabc.edu.br

b e-mail: roldao.rocha@ufabc.edu.br (corresponding author)
}

portional to the trace of the stress-energy-momentum tensor, which vanishes for conformally invariant theories. However, upon quantization, conformal invariance under Weyl rescalings may be broken and conformal anomalies set in [57]. In this case, the trace of the stress-energy-momentum tensor may achieve a non-null expectation value and, thus, a conformal anomaly regards a trace anomaly [58-63]. In the context of the gravitational decoupling procedure, comparing the holographic Weyl anomaly to the trace anomaly of the energy-momentum tensor from 4D field theory leads to a quantity that can probe and measure the source of the gravitational decoupling [64]. Hence, the calculation of the trace anomaly-to-holographic Weyl anomaly ratio makes one capable to place the gravitational decoupling, in the context of three possible metrics describing hairy black holes, as a reliable AdS/CFT realization.

This paper is organized as follows: Sect. 2 is dedicated to reviewing the gravitational decoupling procedure, obtaining three different metrics for gravitational decoupled hairy black holes. In Sect. 3, the trace anomalies are computed for these three solutions, from the point of view of CFT, and compared to the respective values predicted by the AdS/CFT duality. Section 5 is dedicated to conclusions.

\section{Gravitational decoupling and hairy black holes}

The gravitational decoupling procedure can be straightforwardly introduced when kernel solutions of Einstein's effective field equations can be used to decouple any intricate stress-energy-momentum tensor into manageable pieces [2,5], including the case of hairy black holes [3]. When one regards Einstein's field equations,

$G_{\mu \nu}:=R_{\mu \nu}-\frac{1}{2} R g_{\mu \nu}=\kappa^{2} \stackrel{\circ}{T}_{\mu \nu}$ 
where the stress-energy-momentum tensor, satisfying the conservation equation $\nabla_{\mu} \stackrel{\circ}{T}^{\mu \nu}=0$, can be split as

$\stackrel{\circ}{T}_{\mu \nu}=\mathrm{T}_{\mu \nu}+\alpha \Theta_{\mu \nu}$,

for $\mathrm{T}_{\mu \nu}$ being a general-relativistic solution and $\Theta_{\mu \nu}$ encoding additional sources in the gravitational sector, for $\alpha$ being an arbitrary decoupling parameter that is not perturbative, in general. One considers static, spherically symmetric, stellar distributions described by the metric

$d s^{2}=e^{v(r)} d t^{2}-e^{\lambda(r)} d r^{2}-r^{2} d \Omega^{2}$,

where $d \Omega^{2}$ denotes the solid angle element. The Einstein's field equations (1) are equivalently written as

$\kappa^{2}\left(\mathrm{~T}_{0}^{0}+\Theta_{0}^{0}\right)=\frac{1}{r^{2}}-e^{-\lambda}\left(\frac{1}{r^{2}}-\frac{\lambda^{\prime}}{r}\right)$,

$\kappa^{2}\left(\mathrm{~T}_{1}^{1}+\Theta_{1}^{1}\right)=\frac{1}{r^{2}}-e^{-\lambda}\left(\frac{1}{r^{2}}+\frac{v^{\prime}}{r}\right)$,

$\kappa^{2}\left(\mathrm{~T}_{2}^{2}+\Theta_{2}^{2}\right)=-\frac{e^{-\lambda}}{4}\left(2 v^{\prime \prime}+v^{\prime 2}-\lambda^{\prime} v^{\prime}+2 \frac{v^{\prime}-\lambda^{\prime}}{r}\right)$

where the prime denotes the derivative with respect to the variable $r$. Equations (4a-4c) regard the effective density, and the effective radial and tangential pressures, respectively given by $[2,5]$

$$
\begin{gathered}
\stackrel{\circ}{\rho}=\rho+\alpha \Theta_{0}^{0}, \\
\stackrel{\circ}{r}_{r}=p-\alpha \Theta_{1}^{1}, \\
\stackrel{\circ}{p}_{t}=p-\alpha \Theta_{2}^{2},
\end{gathered}
$$

with anisotropy

$\Delta=\stackrel{\circ}{p}_{t}-\stackrel{\circ}{p}_{r}$.

A solution to Einstein's field equations (1) for the single kernel source $\mathrm{T}_{\mu \nu}$ was considered [3,5],

$d s^{2}=e^{\xi(r)} d t^{2}-e^{\mu(r)} d r^{2}-r^{2} d \Omega^{2}$,

where

$e^{-\mu(r)} \equiv 1-\frac{\kappa^{2}}{r} \int_{0}^{r} x^{2} \mathrm{~T}_{0}^{0}(x) d x=1-\frac{2 m(r)}{r}$

is the Misner-Sharp-Hernandez function. The additional source $\Theta_{\mu \nu}$ drives the gravitational decoupling of the kernel metric (7), implemented by the mappings

$$
\begin{aligned}
\xi(r) & \mapsto v(r)=\xi(r)+\alpha g(r) \\
e^{-\mu(r)} & \mapsto e^{-\lambda(r)}=e^{-\mu(r)}+\alpha f(r),
\end{aligned}
$$

where $f(r)[g(r)]$ is the geometric deformation for the radial [temporal] metric component. Eqs. (9a, 9b) split the Einstein's field equations (4a)-(4c) into two distinct arrays. The first one encodes the Einstein's field equations for $\mathrm{T}_{\mu \nu}$, solved by the kernel metric (7). The second one is associated to $\Theta_{\mu \nu}$ and reads

$$
\begin{aligned}
\kappa^{2} \Theta_{0}^{0} & =-\alpha\left(\frac{f}{r^{2}}+\frac{f^{\prime}}{r}\right), \\
\kappa^{2} \Theta_{1}^{1}+\alpha \frac{e^{-\mu} g^{\prime}}{r} & =-\alpha f\left(\frac{1}{r^{2}}+\frac{v^{\prime}}{r}\right) \\
\kappa^{2} \Theta_{2}^{2}+\alpha f\left(2 v^{\prime \prime}+v^{\prime 2}+\frac{2 v^{\prime}}{r}\right) & =-\alpha \frac{f^{\prime}}{4}\left(v^{\prime}+\frac{2}{r}\right)+V
\end{aligned}
$$

where [1]

$V(r)=\alpha e^{-\mu}\left(2 g^{\prime \prime}+g^{\prime 2}+\frac{2 g^{\prime}}{r}+2 \xi^{\prime} g^{\prime}-\mu^{\prime} g^{\prime}\right)$

The tensor-vacuum, defined for $\Theta_{\mu \nu} \neq 0$ and $\mathrm{T}_{\mu \nu}=0$, leads to hairy black hole solutions [65]. Equations (4a)-(4b) then yield a negative radial pressure,

$\stackrel{\circ}{p}_{r}=-\stackrel{\circ}{\rho}$.

and, together to the Schwarzschild solution, it implies that

$\alpha f(r)=\left(1-\frac{2 M}{r}\right)\left(e^{\alpha g(r)}-1\right)$,

so that the line element (3) becomes

$$
\begin{aligned}
d s^{2}= & \left(1-\frac{2 M}{r}\right) e^{\alpha g(r)} d t^{2}-\left(1-\frac{2 M}{r}\right)^{-1} e^{-\alpha g(r)} d r^{2} \\
& -r^{2} d \Omega^{2}
\end{aligned}
$$

In the radial range $r \geq 2 M$, the tensor-vacuum is given by expressing $\Theta_{0}^{0}$ by the most general linear combination of the radial and tangential components of the stress-energymomentum tensor, as

$\Theta_{0}^{0}=a \Theta_{1}^{1}+b \Theta_{2}^{2}$,

with $a, b \in \mathbb{R}$ denoting the coefficients of the linear combination. Equations (10a)-(10c) then yield

$$
\begin{aligned}
& b r(r-2 M) h^{\prime \prime}+2[(a+b-1) r-2(a-1) M] h^{\prime} \\
& \quad+2(a-1) h=2(a-1),
\end{aligned}
$$

for $h(r)=e^{\alpha g(r)}$. A trivial deformation corresponding to the standard Schwarzschild solution can be yielded when $a=1$. The solution of Eq. (16) can be written as

$e^{\alpha g(r)}=1+\frac{1}{r-2 M}\left[\ell_{0}+r\left(\frac{\ell}{r}\right)^{n}\right]$,

where $\ell_{0}=\alpha \ell$ is a primary hair charge, whereas

$n=2(a-1) / b$,

with $n>1$ for asymptotic flatness. 
In the tensor-vacuum background, this line element is produced by the effective density, the radial, and tangential pressures, respectively,

$$
\begin{aligned}
\stackrel{\circ}{\rho} & =\Theta_{0}^{0}=\alpha \frac{(n-1) \ell^{n}}{\kappa^{2} r^{n+2}}, \\
\stackrel{\circ}{p}_{r} & =-\Theta_{1}^{1}=-\stackrel{\circ}{\rho}, \\
\stackrel{\circ}{p}_{t} & =-\Theta_{2}^{2}=\frac{n}{2} \stackrel{\circ}{\rho} .
\end{aligned}
$$

On the other hand, the dominant energy conditions,

$\stackrel{\circ}{\rho} \geq\left|\stackrel{\circ}{p}_{r}\right|, \quad \stackrel{\circ}{\rho} \geq\left|\stackrel{\circ}{p}_{t}\right|$,

yield $n \leq 2[2,3,5]$. Besides, the strong energy conditions,

$$
\begin{aligned}
& \stackrel{\circ}{\rho}+\stackrel{\circ}{p}_{r}+2 \stackrel{\circ}{p}_{t} \geq 0, \\
& \stackrel{\circ}{\rho}+\stackrel{\circ}{p}_{r} \geq 0, \\
& \stackrel{\circ}{\rho}+\stackrel{\circ}{p}_{t} \geq 0,
\end{aligned}
$$

make Eq. (12) to read

$$
-\Theta_{0}^{0} \leq \Theta_{2}^{2} \leq 0 \text {. }
$$

Therefore, together with Eqs. (10a) and (10c), Eq. (22) can be written as

$$
\begin{aligned}
& G_{1}(r):=h^{\prime \prime}(r-2 M)+2 h^{\prime} \geq 0, \\
& G_{2}(r):=h^{\prime \prime} r(r-2 M)+4 h^{\prime} M-2 h+2 \geq 0 .
\end{aligned}
$$

The mapping

$h(r) \mapsto h(r)-\frac{\ell_{0}}{r-2 M}$

leaves $G_{1}(r)$ and $G_{2}(r)$ invariant. Solutions with a proper horizon at $r \sim 2 M$, which also behave approximately like the Schwarzschild metric for $r \gg 2 M$, yield $G_{1}(r)=0$. Hence, solving Eq. (23a) implies that

$h(r)=c_{1}-\alpha \frac{\ell-r e^{-r / M}}{r-2 M}$.

Also, Eq. (25) is also constrained to (23b). Replacing (25) in (14) implies the metric

$e^{v}=e^{-\lambda}=1-\frac{2 \mathcal{M}}{r}+\alpha e^{-r /(\mathcal{M}-\alpha \ell / 2)}$,

to represent a hairy black hole, where $\mathcal{M}=M+\alpha \ell / 2$.

Now, the strong energy conditions are consistent with $\ell \geq$ $2 M / e^{2}$, whose extremal case $\ell=2 M / e^{2}$ leads to

$e^{\nu}=e^{-\lambda}=1-\frac{2 M}{r}+\alpha\left(e^{-r / M}-\frac{2 M}{e^{2} r}\right)$.

which has the horizon at $r_{\mathrm{HOR}}=2 M$. The dominant energy conditions,

$\stackrel{\circ}{\rho} \geq\left|\stackrel{\circ}{r}_{r}\right|$,

$\stackrel{\circ}{\rho} \geq\left|\dot{p}_{t}\right|$, in terms of (5a) and (5c) are respectively equivalent to

$$
\begin{array}{r}
-r(r-2 M) h^{\prime \prime}-4(r-M) h^{\prime}-2 h+2 \geq 0, \\
r(r-2 M) h^{\prime \prime}+4 M h^{\prime}-2 h+2 \geq 0 .
\end{array}
$$

Solving (29a) for $r \sim 2 M$ and $r \gg M$ yields [65]

$h(r)=1-\frac{1}{r-2 M}\left(\alpha \ell+\alpha M e^{-r / M}-\frac{Q^{2}}{r}\right)$,

where the charge $Q=Q(\alpha)$ encompasses also tidal charges generated by additional gravitational sectors. Equation (30) also has to satisfy (29b), which reads

$\frac{4 Q^{2}}{r^{2}} \geq \frac{\alpha}{M}(r+2 M) e^{-r / M}$.

Using (30) into the line element (14), yields

$e^{\nu}=e^{-\lambda}=1-\frac{2 M+\alpha \ell}{r}+\frac{Q^{2}}{r^{2}}-\frac{\alpha M e^{-r / M}}{r}$,

such that

$\stackrel{\circ}{\rho}=\Theta_{0}^{0}=-\stackrel{\circ}{p}_{r}=\frac{Q^{2}}{\mathrm{~K}^{2} r^{4}}-\frac{\alpha e^{-r / M}}{\mathrm{~K}^{2} r^{2}}$

The metric (32) also represents hairy black holes, where $Q$ and $\ell_{0}=\alpha \ell$ comprise charges generating primary hair.

The horizon radii $r_{\mathrm{HOR}}$ are given by solutions of

$\alpha \ell=r_{\mathrm{HOR}}-2 M+\frac{Q^{2}}{r_{\mathrm{HOR}}}-\alpha M e^{-r_{\mathrm{HOR}} / M}$,

which allows us to write the metric functions (32) as

$$
\begin{aligned}
e^{v}= & e^{-\lambda}=1-\frac{r_{\mathrm{HOR}}}{r}\left(1+\frac{Q^{2}}{r_{\mathrm{HOR}}^{2}}-\frac{\alpha M}{r_{\mathrm{HOR}}} e^{-r_{\mathrm{HOR}} / M}\right) \\
& +\frac{Q^{2}}{r^{2}}-\frac{\alpha M}{r} e^{-r / M} .
\end{aligned}
$$

To find analytical solutions to $r_{\mathrm{HOR}}$, appropriate values of the parameters $\alpha, Q$, and $\ell$ must be chosen. However, since the dominant energy conditions demand $r_{\mathrm{HOR}} \geq 2 \mathrm{M}$, the choice of these values cannot be arbitrary. Evaluating the effective density (33) at the event horizon, and making use of Eq. (34) imply that

$Q^{2} \geq \frac{4 \alpha M^{2}}{e^{2}}, \quad \ell \geq \frac{M}{e^{2}}$.

The physical interpretation of $Q$ encompasses the case of an electric charge, but not only restricted to it, but also encoding the possibility of hidden gauge charges, tidal charge, and eventually, Kaluza-Klein stringy effects [34], or any other source. In the case where $Q$ represents an electric charge, the electrovacuum generated by the Reissner-Nordström solution additionally accommodates a tensor-vacuum that is proportional to $\alpha$ in Eq. (33). One must emphasize that the Reissner-Nordström metric has an event horizon

$r_{\mathrm{RN}}=M+\sqrt{M^{2}-Q^{2}}<2 M$, 
and also an inner Cauchy horizon,

$r_{\mathrm{C}} \equiv M-\sqrt{M^{2}-Q^{2}}<r_{\mathrm{HOR}}$.

The solution (32) can thus yield three ramifications wherein the event horizon $r_{\mathrm{HOR}}$ has straightforward analytical formulæ and the dominant energy conditions are satisfied. Similarly to the Reissner-Nordström solution, the three cases to be studied have an inner Cauchy horizon $r_{\mathrm{C}}<r_{\text {нов }}$.

If the event horizon is made proportional to the mass, as $r_{\text {HоR }}=k M$, as long as $k \geq 2$, to satisfy the dominant energy conditions, the metric components are given by

$e^{\nu}=e^{-\lambda}=1-\frac{2 \mathcal{M}}{r}+\frac{Q^{2}}{r^{2}}-\frac{\alpha r_{\mathrm{HOR}}}{k r} e^{-k r / r_{\mathrm{HOR}}}$,

where the Reissner-Nordström-like event horizon reads

$r_{\text {HOR }}=\tilde{\mathcal{M}}+\sqrt{\tilde{\mathcal{M}}^{2}-\tilde{Q}^{2}}$,

where $\tilde{\mathcal{M}}=\mathcal{M} / \beta$ and $\tilde{Q}^{2}=Q^{2} / \beta$ with

$\beta=1-\alpha \frac{e^{-k}}{k}$.

Therefore the metric components (39) describe a black hole solution arising from nonlinear electrodynamics, with event horizon

$r_{\mathrm{HOR}}=\frac{r_{\mathrm{RN}}}{\beta} \geq r_{\mathrm{RN}}$,

as $\beta \leq 1$. The nonlinear electrodynamics is obtained when one identifies

$\Theta_{\mu \nu}=-\mathcal{L}(F) g_{\mu \nu}-\mathcal{L}_{F} F_{\mu}^{\rho} F_{\rho \nu}$,

where

$F=\frac{1}{4} F_{\rho \sigma} F^{\rho \sigma}, \quad \mathcal{L}_{\mathrm{F}}=\frac{\partial \mathcal{L}}{\partial F}$

When static, spherically symmetric, stellar distributions described by the metric (3) are regarded, the field strength reads

$F_{\mu \nu}(r)=\left(\delta_{\mu}^{0} \delta_{v}^{1}-\delta_{\mu}^{1} \delta_{\nu}^{0}\right) E(r)$,

where the electric field is given by

$E(r)=\frac{Q}{r^{2}}-\frac{\alpha e^{-\frac{k r}{r_{\mathrm{HOR}}}}}{4 r_{\mathrm{HOR}} Q}\left(k r+2 r_{\mathrm{HOR}}\right)$.

Introducing the field

$P=\mathcal{L}_{\mathrm{F}}^{2} F_{\rho \sigma} F^{\rho \sigma}$,

the underlying nonlinear electrodynamics can be thus placed into the $P$-dual framework $[1,66]$, described by the Lagrangian

$\mathcal{L}(P)=-4 \pi P-\frac{\alpha k(-2 P)^{\frac{1}{4}}}{4 \sqrt{\pi Q} r_{\mathrm{HOR}}} \exp \left[\frac{k \sqrt{Q}}{2 \sqrt{\pi}(-2 P)^{\frac{1}{4}} r_{\mathrm{HOR}}}\right]$, where

$\mathfrak{G}(P)=-\frac{k \sqrt{Q}}{2 \sqrt{\pi} r_{\mathrm{HOR}}(-2 P)^{\frac{1}{4}}}$.

One can read off the relation $Q^{2} \sim \alpha$, yielding the Schwarzschild standard solution whenever $\alpha \rightarrow 0$. When $Q$ represents an electric charge, one can state that the ReissnerNordström electrovacuum is permeated by a tensor-vacuum governed by (48).

As concrete examples, one can saturate the inequalities (36), and (35) become, defining $\mathrm{M}=M\left(1+\frac{\alpha}{2 e^{2}}\right)$,

$e^{v_{(\mathrm{I})}}=e^{-\lambda_{(\mathrm{I})}}=1-\frac{2 \mathrm{M}}{r}+\frac{Q^{2}}{r^{2}}-\frac{\sqrt{\alpha} Q}{2 r} e^{1-2 \sqrt{\alpha} r / e Q}$

which can be interpreted as a nonlinear electrodynamics coupled with gravity, similarly to the content in the last paragraph [3]. The event horizons are placed at $r_{\text {нов }}=2 M$, and $r_{\mathrm{HOR}}=\frac{e}{\sqrt{\alpha}} Q$.

In the second case, the relation

$Q^{2}=\alpha \ell M\left(2+\alpha e^{-\alpha \ell / M}\right)$,

leads to

$$
\begin{gathered}
e^{v_{(\mathrm{II})}=} e^{-\lambda_{(\mathrm{II})}}=1-\frac{2 M+\alpha \ell}{r}+\frac{2 \alpha \ell M}{r^{2}} \\
-\frac{\alpha M}{r^{2}} e^{-r / M}\left(r-\alpha \ell e^{\frac{r-\alpha \ell}{M}}\right) .
\end{gathered}
$$

The event horizon is now at $r_{\mathrm{HOR}}=\alpha \ell \geq 2 M$. As $\alpha \ell \sim$ $M$, Eq. (52) can be also realized as a solution in nonlinear electrodynamics coupled with gravity.

Finally, when

$Q^{2}=\alpha M(2 M+\alpha \ell) e^{-\frac{(2 M+\alpha \ell)}{M}}$,

the metric components $e^{v_{(\text {III })}}=e^{-\lambda_{\text {(III) }}}$ read

$$
\begin{aligned}
e^{v_{(I I I)}}= & 1-\frac{\alpha M}{r^{2}} e^{-r / M}\left[r-(2 M+\alpha \ell) e^{\frac{r-(2 M+\alpha \ell)}{M}}\right] \\
& -\frac{2 M+\alpha \ell}{r} .
\end{aligned}
$$

The event horizon is at $r_{\mathrm{HOR}}=2 M+\alpha \ell=2 \mathcal{M} \geq 2 M$.

\section{Weyl and trace anomalies of gravitational decoupled hairy black holes}

The holographic Weyl anomaly is reminiscent of the regularization process applied to the gravitational part of the action under conformal transformations [59]. In general, the anomaly can be expressed by

$\mathcal{A} \propto E_{(d)}+I_{(d)}$, 
where $E_{(d)}$ is the $d$-dimensional Euler density, and $I_{(d)}$ denotes a conformal invariant. ${ }^{1}$ In four dimensions the Euler density takes the form

$E_{(4)}=\frac{1}{64}\left(K-4 R^{\mu v} R_{\mu v}+R^{2}\right)$.

Up to a multiplicative constant, the holographic Weyl anomaly becomes

$$
\langle T\rangle_{\mathrm{CFT}} \sim\left(R^{\mu \nu} R_{\mu \nu}-\frac{1}{3} R^{2}\right) .
$$

On the other hand, the trace anomaly is a function of the matter content on a curved background together with its geometric aspects [67]

$$
\langle T\rangle_{4 \mathrm{D}} \sim\left(K-R^{\mu v} R_{\mu \nu}-\square R\right) .
$$

This anomaly quantifies the deviation from conformal invariance, i. e., the vanishing of this particular quantity indicates that the associated dual theory preserves conformal symmetry.

Trace anomalies from the field theory side can be compared to the one found in the CFT using the coefficient [64]

$\Gamma=\left|1-\frac{\langle T\rangle_{4 \mathrm{D}}}{\langle T\rangle_{\mathrm{CFT}}}\right|$,

where the definitions given in Eqs. $(57,58)$ have been applied. The quantity $K=R_{\mu \nu \rho \sigma} R^{\mu \nu \rho \sigma}$ denotes the Kretschmann scalar $K$. This result holds in the context of asymptotic AdS backgrounds [59] and here we discuss the possibility of emulating this result to the gravitational decoupling of hairy black holes.

The coefficient (59) can quantify how AdS/CFT is reliable in the context where the metrics $(50,52,54)$ of gravitational decoupled hairy black holes are taken into account. It measures the trace anomalies associated with them and how the additional sources backreact, in the gravitational decoupling setup. The coefficient $\Gamma$ can formally run from 0 to infinity, and AdS/CFT can underlie this setup for values that are close to unit $[64,68]$. In fact, for the Schwarzschild case, $\langle T\rangle_{4 \mathrm{D}} \propto K$ and $\langle T\rangle_{\mathrm{CFT}}=0$ yielding $\Gamma \rightarrow \infty$, what compromises AdS/CFT in the general-relativistic case [64].

On braneworld scenarios, one seeks for spacetimes where $\Gamma \ll 1$, where the quantum conformal field theory on the brane and the classical gravity on the bulk descriptions are dual and equivalent $[64,69]$. The case when $\Gamma=1$ was obtained evaluating the coefficient (59) for a braneworld black hole solution [64]. One expects other solutions to be more intricate, as in the case of gravitational decoupled hairy black holes $(50,52,54)$.

\footnotetext{
${ }^{1}$ For $d=4$ this invariant is unique, being given by the contraction of the Weyl tensor to itself [59].
}

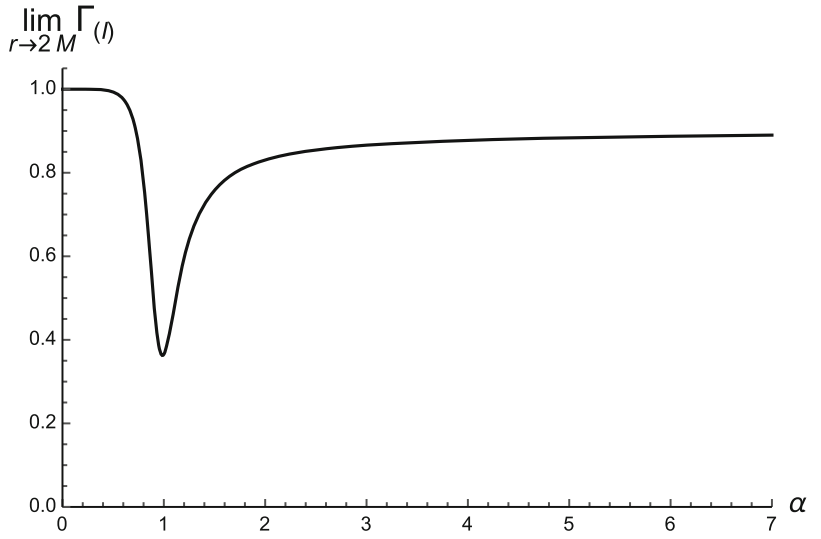

Fig. 1 Plot of $\lim _{r \rightarrow 2 M} \Gamma_{\text {(I) }}$ as a function of the decoupling parameter $\alpha$

Notice that for large values of $r$, all three coefficients have near unit values,

$$
\begin{aligned}
& \lim _{r \rightarrow \infty} \Gamma^{(\mathrm{I})} \approx 1, \\
& \lim _{r \rightarrow \infty} \Gamma^{(\mathrm{II})} \approx 1, \\
& \lim _{r \rightarrow \infty} \Gamma^{(\mathrm{III})} \approx 1 .
\end{aligned}
$$

This is interesting and relevant, as such behavior is different of the Schwarzschild kernel metric used to derive these solutions using the gravitational decoupling method. Next, the limiting expressions for $r \rightarrow 2 M$ are analyzed in Figs. 1, 2 and 3, which display $\left.\Gamma\right|_{r \rightarrow 2 M}$ as a function of $\alpha$. Since $\alpha$ is not a perturbation parameter, at least technically it can assume any value. Thus the coefficient $\Gamma_{\mathrm{CFT}}$ can be determined in the $\alpha \rightarrow \infty$ limit,

$$
\begin{aligned}
& \lim _{\substack{\alpha \rightarrow \infty \\
r \rightarrow 2 M}} \Gamma_{\text {(I) }}=0.923-\mathcal{O}\left(\frac{1}{\alpha}\right), \\
& \lim _{\substack{\alpha \rightarrow \infty \\
r \rightarrow 2 M}} \Gamma_{(\mathrm{II})}=1+\mathcal{O}\left(\frac{1}{\alpha}\right), \\
& \lim _{\substack{\alpha \rightarrow \infty \\
r \rightarrow 2 M}} \Gamma_{\text {(III) }}=0 .
\end{aligned}
$$

Considering these limits along with the plots in Figs. 1, 2 and 3 , one can safely state that $\Gamma \leq 1$ at the regions of interest, namely, for the concomitant limits $r \rightarrow \infty$ and $r \rightarrow 2 M$.

In Figs. 1 and 2 one can clearly see a bump, where the value of $\Gamma$ is minimum. These values are $\Gamma_{\text {(I) }} \approx 0.367$, when $\alpha \approx 0.988$, as $\Gamma_{\text {(II) }} \approx 0$, for $\alpha \approx 0.202$. In fact, the tiny range $0.2015<\alpha<0.2032$ yields $\Gamma_{\text {(II) }}<3 \times 10^{-5}$.

As the limiting values of $\Gamma$ for $r \rightarrow \infty$ do not depend on the parameter $\alpha$, we can use the values mentioned above on the metrics to conclude that, if the AdS/CFT correspondence holds for these particular solutions obtained via gravitational decoupling, then the best agreement between classical gravity and the associated field theory is then implemented. 


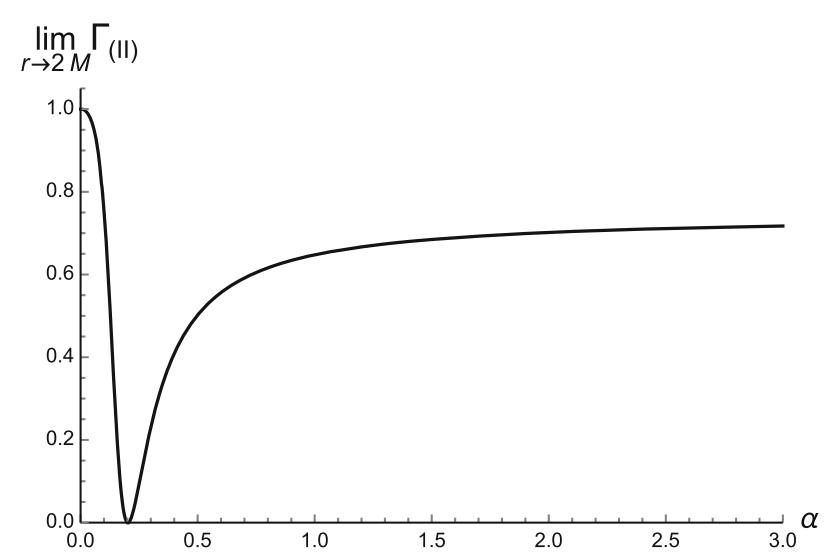

Fig. 2 Plot of $\lim _{r \rightarrow 2 M} \Gamma_{\text {(II) }}$ as a function of the decoupling parameter $\alpha$

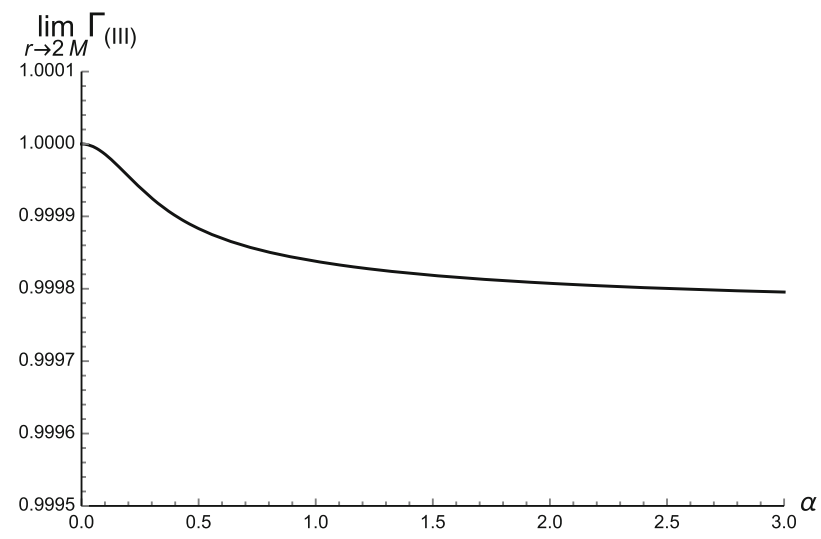

Fig. 3 Plot of $\lim _{r \rightarrow 2 M} \Gamma_{\text {(III) }}$ as a function of the decoupling parameter $\alpha$

\section{Relation to AdS/CFT correspondence}

Having these results, it is important to point out how the solutions can be used in the context of AdS/CFT correspondence, given the compliance between gravity and the associated boundary theory from the computation of $\Gamma$. For this connection to be established, one recalls Einstein's equations in its full form

$R_{\mu \nu}-\frac{1}{2} R g_{\mu \nu}=\Lambda_{4} g_{\mu \nu}+\bar{T}_{\mu \nu}$

where $\Lambda_{4}$ is the cosmological constant in 4-dimensions, and $\bar{T}_{\mu \nu}$ is the energy momentum tensor describing all other fields except the cosmological constant one. In Sect. 2 the solutions studied were derived requiring that $T_{\mu \nu}=0$ and $\Theta_{\mu \nu} \neq 0$. From the explicit expressions of the solutions, c.f. Eqs. $(50,52,54)$, one can check that the limit $r \mapsto \infty$ leads to the Minkowski spacetime. However, the MGD has a strong connection to brane-world scenarios [70], which can be employed to establish the connection.
In the brane-world setup, the stress-energy-momentum tensor associated to the brane has the most general form [71]

$\bar{T}_{\mu \nu}=T_{\mu \nu}+E_{\mu \nu}+\gamma^{-1} S_{\mu \nu}+L_{\mu \nu}+P_{\mu \nu}$,

where $\gamma$ denotes the brane tension. The first term is the ordinary energy-momentum tensor from Einstein's equations, as already pointed out $T_{\mu \nu}=0$. The third term contains corrections of second order on the energy-momentum tensor $T_{\mu \nu}$, such that $S_{\mu \nu} \propto \mathcal{O}\left(T_{\mu \nu}^{2}\right)$, and therefore vanishes as well. The remaining terms in Eq. (67) carry non-local effects and also affect the energy-momentum tensor depending on the geometric procedure one uses to embed the brane in the bulk. Specifically, $L_{\mu \nu}$ accounts for the embedding, and is associated with the bending of the brane concerning the codimension- 1 bulk. $P_{\mu \nu}$ contains possible stringy effects living in the bulk. $E_{\mu \nu}$ describes a Weyl fluid in the bulk and is responsible for non-local effects and anisotropies. Explicit expressions for these terms can be found in [71,72].

Notice that this description is valid on a context where the solutions describe a brane, which is embedded in a higher dimensional space-time, and therefore the quantities appearing on (67) are related to the higher dimensional bulk. It is important to remark that the bulk is governed by its own Einstein's equations, such as Eq. (66) in one extra dimension, where other matter fields can be defined, and it has its own cosmological constant. The cosmological constants in the bulk and on the brane are related to each other by fine tuning with the brane tension $\gamma,[73]$

$\Lambda_{4}=\frac{\kappa_{5}^{2}}{2}\left(\Lambda_{5}+\frac{1}{6} \kappa_{5}^{2} \gamma^{2}\right)$,

where $\kappa_{5}=8 \pi G_{5}$ and $G_{5}$ is the Newton constant in five dimensions, which is related to the 4 dimensional Newton constant by the Planck length $\ell_{p}$ as $G_{5}=\ell_{p} G_{4}$. The brane tension cannot be arbitrarily defined and has a predicted lower bound for its value $\gamma \geq 2.8131 \times 10^{-6}$ [33]. To prevent matter fields living in the bulk to interact with matter fields in the brane one has to fine tune the cosmological constant in Eq. (68) such that $\Lambda_{4}=0$ [10]. Given the lower bound on the brane tension, one finds immediately that

$\Lambda_{5}=-\frac{1}{6} \kappa_{5}^{2} \gamma^{2}$

Implying that the bulk where the brane is located is an AdS space-time. Considering the overall setup described in Sect. 2, one can therefore identify $\bar{T}_{\mu \nu}=\alpha \Theta_{\mu \nu}$, therefore

$\alpha \Theta_{\mu \nu}=E_{\mu \nu}+L_{\mu \nu}+P_{\mu \nu}$.

From this connection, the AdS/CFT conjecture can be applied to the described metrics following the prescription of [74]. Reference [1] established the way how the general gravitational decoupling can be led to the membrane paradigm of AdS/CFT. 


\section{Conclusions}

The gravitational decoupling of hairy black holes was utilized and inspected with the apparatus provided by trace and Weyl anomalies. The gravitational decoupling was shown to be a trustworthy model, in the context of AdS/CFT. Since the value of the $\Gamma$ coefficient, for the gravitational decoupling case, was shown to be near the unit, it means that the gravitational decoupling solutions may occupy a privileged place and can play a prominent role in emulating AdS/CFT on gravitational decoupled solutions. The $\alpha \rightarrow \infty$ limit in Eqs. (63-65) can be seen as a regime where the stress-energy-momentum tensor (2) has only the additional source contribution, in the gravitational sector, being the general-relativistic source negligible. This characterizes, in fact, the tensor vacuum regime. The coefficient (59) quantifies the excitation of gravitationally decoupled matter fields and estimates the signatures of gravitational waves beyond the general-relativistic setup, measuring fluctuations of the decoupled source. Hence, the anomaly coefficient (59) brings useful information about placing gravitational decoupled hairy black holes in the AdS/CFT framework, implementing a method to quantify trace anomalies in this context, besides also quantifying backreaction of gravitationally decoupled additional sources, driven by the parameter $\alpha$.

Acknowledgements PM thanks Coordenação de Aperfeiçoamento de Pessoal de Nível Superior-Brasil (CAPES)-Finance Code 001. RdR is grateful to FAPESP (Grant No. 2017/18897-8 and No. 2021/01089-1) and the National Council for Scientific and Technological Development-CNPq (Grants No. 303390/2019-0 and No. 406134/2018-9, and No. 402535/2021-9, for partial financial support.

Data Availability Statement This manuscript has no associated data or the data will not be deposited. [Authors' comment: This is a theoretical study and no experimental data has been listed.]

Open Access This article is licensed under a Creative Commons Attribution 4.0 International License, which permits use, sharing, adaptation, distribution and reproduction in any medium or format, as long as you give appropriate credit to the original author(s) and the source, provide a link to the Creative Commons licence, and indicate if changes were made. The images or other third party material in this article are included in the article's Creative Commons licence, unless indicated otherwise in a credit line to the material. If material is not included in the article's Creative Commons licence and your intended use is not permitted by statutory regulation or exceeds the permitted use, you will need to obtain permission directly from the copyright holder. To view a copy of this licence, visit http://creativecomm ons.org/licenses/by/4.0/.

Funded by SCOAP ${ }^{3}$.

\section{References}

1. J. Ovalle, Phys. Rev. D 95, 104019 (2017). arXiv:1704.05899

2. J. Ovalle, Phys. Lett. B 788, 213-218 (2019). arXiv:1812.03000
3. J. Ovalle, R. Casadio, E. Contreras, A. Sotomayor, Phys. Dark Univ. 31, 100744 (2021). arXiv:2006.06735

4. R. Casadio, J. Ovalle, Gen. Relativ. Gravit. 46, 1669 (2014). arXiv:1212.0409

5. J. Ovalle, R. Casadio, R. da Rocha, A. Sotomayor, Eur. Phys. J C78, 122 (2018). arXiv:1708.00407

6. I. Antoniadis, N. Arkani-Hamed, S. Dimopoulos, G.R. Dvali, Phys. Lett. B 436, 257 (1998). arXiv:9804398

7. J. Ovalle, L.A. Gergely, R. Casadio, Class. Quantum Gravity 32, 045015 (2015). arXiv: 1405.0252

8. J. Ovalle, R. Casadio, A. Sotomayor, Adv. High Energy Phys. 2017, 9756914 (2017). arXiv: 1612.07926

9. R. Casadio, J. Ovalle, R. da Rocha, Class. Quantum Gravity 31, 045016 (2014). arXiv: 1310.5853

10. J. Ovalle, F. Linares, A. Pasqua, A. Sotomayor, Class. Quantum Gravity 30, 175019 (2013). arXiv:1304.5995

11. J. Ovalle, F. Linares, Phys. Rev. D 88, 104026 (2013). arXiv: 1311.1844

12. J. Ovalle, A. Sotomayor, Eur. Phys. J. Plus 133, 428 (2018). arXiv: 1811.01300

13. R. Casadio, J. Ovalle, Phys. Lett. B 715, 251-255 (2012). arXiv: 1201.6145

14. R. Casadio, J. Ovalle, R. da Rocha, EPL 110, 40003 (2015). arXiv: 1503.02316

15. R. Casadio, R. da Rocha, Phys. Lett. B 763, 434-438 (2016). arXiv: 1610.01572

16. R. da Rocha, Symmetry 12, 508 (2020). arXiv:2002.10972

17. A. Fernandes-Silva, R. da Rocha, Eur. Phys. J. C 78, 271 (2018). arXiv: 1708.08686

18. E. Contreras, Eur. Phys. J. C 78, 678 (2018). arXiv: 1807.03252

19. J. Ovalle, Mod. Phys. Lett. A 23, 3247-3263 (2008). arXiv:gr-qc/0703095

20. M. Sharif, S. Saba, Eur. Phys. J. C 78, 921 (2018). arXiv: 1811.08112

21. M. Sharif, S. Sadiq, Chin. J. Phys. 60, 279-289 (2019)

22. E. Morales, F. Tello-Ortiz, Eur. Phys. J. C 78, 841 (2018). arXiv:1808.01699

23. A. Rincón, L. Gabbanelli, E. Contreras, F. Tello-Ortiz, Eur. Phys. J. C 79, 873 (2019). arXiv: 1909.00500

24. S. Hensh, Z. Stuchlik, Eur. Phys. J. C 79, 834 (2019). arXiv: 1906.08368

25. J. Ovalle, C. Posada, Z. Stuchlík, Class. Quantum Gravity 36, 205010 (2019). arXiv:1905.12452

26. L. Gabbanelli, J. Ovalle, A. Sotomayor, Z. Stuchlik, R. Casadio, Eur. Phys. J. C 79, 486 (2019). arXiv: 1905.10162

27. F. Tello-Ortiz, Eur. Phys. J. C 80, 413 (2020)

28. L. Gabbanelli, A. Rincón, C. Rubio, Eur. Phys. J. C 78, 370 (2018). arXiv: 1802.08000

29. G. Panotopoulos, A. Rincón, Eur. Phys. J. C 78, 851 (2018). arXiv: 1810.08830

30. C.L. Heras, P. Leon, Fortsch. Phys. 66, 1800036 (2018). arXiv: 1804.06874

31. E. Contreras, P. Bargueño, Eur. Phys. J. C 78, 558 (2018). arXiv:1805.10565

32. F. Tello-Ortiz, S. Maurya, Y. Gomez-Leyton, Eur. Phys. J. C 80, $324(2020)$

33. A. Fernandes-Silva, A.J. Ferreira-Martins, R. da Rocha, Phys. Lett. B 791, 323-330 (2019). arXiv: 1901.07492

34. R. da Rocha, Phys. Rev. D 95, 124017 (2017). arXiv:1701.00761

35. A. Fernandes-Silva, A.J. Ferreira-Martins, R. da Rocha, Eur. Phys. J. C 78, 631 (2018). arXiv:1803.03336

36. S. Maurya, S. Maharaj, D. Deb, Eur. Phys. J. C 79, 170 (2019)

37. R. Pérez Graterol, Eur. Phys. J. Plus 133, 244 (2018)

38. E. Morales, F. Tello-Ortiz, Eur. Phys. J. C 78, 618 (2018). arXiv:1805.00592 
39. E. Contreras, A. Rincón, P. Bargueño, Eur. Phys. J. C 79, 216 (2019). arXiv: 1902.02033

40. E. Contreras, Class. Quantum Gravity 36, 095004 (2019). arXiv:1901.00231

41. K. Singh, S. Maurya, M. Jasim, F. Rahaman, Eur. Phys. J. C 79, $851(2019)$

42. F. Tello-Ortiz, S.K. Maurya, A. Errehymy, K. Singh, M. Daoud, Eur. Phys. J. C 79, 885 (2019)

43. S. Maurya, F. Tello-Ortiz, Phys. Dark Univ. 29, 100577 (2020). arXiv:1907.13456

44. F.X. Linares Cedeno, E. Contreras, Phys. Dark Univ. 28, 100543 (2020). arXiv:1907.04892

45. M. Sharif, S. Sadiq, Eur. Phys. J. C 78, 410 (2018). arXiv: 1804.09616

46. M. Estrada, F. Tello-Ortiz, Eur. Phys. J. Plus 133, 453 (2018). arXiv: 1803.02344

47. V. Torres-Sanchez, E. Contreras, Eur. Phys. J. C 79, 829 (2019). arXiv: 1908.08194

48. G. Abellán, A. Rincon, E. Fuenmayor, E. Contreras, (2020). arXiv:2001.07961 (Preprint)

49. M. Estrada, R. Prado, Eur. Phys. J. Plus 134, 168 (2019). arXiv:1809.03591

50. P. León, A. Sotomayor, Fortsch. Phys. 67, 1900077 (2019). arXiv:1907.11763

51. R. Casadio, E. Contreras, J. Ovalle, A. Sotomayor, Z. Stuchlick, Eur. Phys. J. C 79, 826 (2019). arXiv:1909.01902

52. M. Sharif, A. Waseem, Ann. Phys. 405, 14-28 (2019)

53. G. Abellan, V. Torres-Sanchez, E. Fuenmayor, E. Contreras, Eur. Phys. J. C 80, 177 (2020). arXiv:2001.08573

54. A. Rincón, E. Contreras, F. Tello-Ortiz, P. Bargueno, G. Abellán, Eur. Phys. J. C 80, 490 (2020). arXiv:2005.10991

55. M. Sharif, A. Majid, Phys. Dark Univ. 30, 100610 (2020). arXiv:2006.04578

56. S. Maurya, K.N. Singh, B. Dayanandan, Eur. Phys. J. C 80, 448 (2020)

57. D. Capper, M. Duff, Nucl. Phys. B 82, 147 (1974)
58. M. Duff, Class. Quantum Gravity 11, 1387-1404 (1994). arXiv:hep-th/9308075

59. M. Henningson, K. Skenderis, JHEP 07, 023 (1998). arXiv:hep-th/9806087

60. I. Kuntz, Eur. Phys. J. C 78, 3 (2018). arXiv: 1712.06582

61. L. Bonora, P. Cotta-Ramusino, C. Reina, Phys. Lett. B 126, 305308 (1983)

62. L. Bonora, S. Giaccari, B. Lima de Souza, JHEP 07, 117 (2014). arXiv: 1403.2606

63. I. Kuntz, R. da Rocha, Nucl. Phys. B 961, 115265 (2020). arXiv:1909.10121

64. R. Casadio, Phys. Rev. D 69, 084025 (2004). arXiv:hep-th/0302171

65. J. Ovalle, R. Casadio, R. da Rocha, A. Sotomayor, Z. Stuchlik, Eur. Phys. J. C 78, 960 (2018). arXiv: 1804.03468

66. I. Salazar, A. Garcia, J. Plebanski, J. Math. Phys. 28, 2171-2181 (1987)

67. N. Birrell, P. Davies, Quantum fields in curved space, Cambridge monographs on mathematical physics (Cambridge Univ. Press, Cambridge, 1984) (ISBN 978-0-521-27858-4, 978-0-521-278584)

68. P. Meert, R. da Rocha, Nucl. Phys. B 967, 115420 (2021). arXiv:2006.02564

69. T. Shiromizu, T. Torii, D. Ida, JHEP 03, 007 (2002). arXiv:hep-th/0105256

70. J. Ovalle, Braneworld stars: anisotropy minimally projected onto the brane, in 9th Asia-Pacific international conference on gravitation and astrophysics (ICGA 9) Wuhan, China, June 28-July 2, 2009, pp 173-182 (2009) arXiv:0909.0531

71. T. Shiromizu, K.I Maeda, M. Sasaki, Phys. Rev. D 62, 024012 (2000)

72. R. Maartens, K. Koyama, Living Rev. Relativ. 13, 5 (2010)

73. L. Randall, R. Sundrum, Phys. Rev. Lett. 83, 3370-3373 (1999). arXiv:hep-ph/9905221

74. J. Soda, Lect. Notes Phys. 828, 235-270 (2011). arXiv:1001.1011 